\title{
Quadratic-like Stability of Nonlinear Homogeneous Systems
}

\author{
Andrey Polyakov
}

\begin{abstract}
A necessary and sufficient condition of stability (stabilizability) of nonlinear homogeneous (control) system is obtained using a topological equivalence of asymptotically stable homogeneous system to a quadratically stable one.
\end{abstract}

\section{INTRODUCTION}

Symmetry is one of well-known properties of physical systems [1]. A type of symmetry of non-linear systems studied in control theory is known as homogeneity [2], [3], [4], [5], [6]. The standard homogeneity was introduced by L. Euler in 17 th century as the symmetry of a mathematical object $f$ (e.g. function, vector field, operator, etc) with respect to the uniform dilation of the argument $x \rightarrow \lambda x$, namely, $f(\lambda x)=\lambda f(x), \lambda>0$. Type of homogeneity is basically identified by dilation. For example, in [7], [8] the uniform dilation is utilized, while the papers [2], [9], [10], [11] deal with the so-called weighted dilation.

Nonlinear homogeneous differential equations/inclusions form an important class of control systems models [4], [12], [13], [14], [15]. They appear as local approximations [3] or set-valued extensions [11] of nonlinear systems and include models of process control [16], nonholonomic systems [17], mechanical models with frictions [10], etc.

The generalized homogeneity (to be studied in this paper) was introduced originally in [18] for infinite dimensional models such as partial differential equations (PDEs). It considers a strongly continuous group of linear bounded operators as a dilation in a Banach space. A lot of wellknown PDEs are homogeneous in generalized sense, e.g. heat, wave, Navier-Stocks, Saint-Venant, Korteweg-de Vries, fast diffusion equations. This paper deals with the finitedimensional models of generalized homogeneous systems represented by ordinary differential equations (ODEs). It is worth stressing that all standard and weighted homogeneous systems are particular cases of the generalized homogeneous ones. Geometric homogeneity [19], [5], [4] is more general type of symmetry allowing dilation groups to be nonlinear.

Stabilization of nonlinear plant is one of classical control problems that is hard to solve constructively in general case, so particular solutions are still of the interest [20], [21]. Stability and stabilizability problems were studied for both standard [2], [8] and weighted homogeneous [9], [22], [23], [6], [24], [25] systems which are the most popular today [10], [11], [26], [13], [15].

This paper deals with quadratic stability problem for nonlinear generalized homogeneous (control) systems. To the best of author's knowledge, the issue of quadratic stability

A. Polyakov is with Inria Lille, 40. av Halley, Villenueve d'Ascq, France, (e-mail: andrey.polyakov@inria.fr) (existence of quadratic Lyapunov function) has not been treated before for the generalized homogeneous systems. Quadratic stabilizability is useful property for control design, since the control tuning procedures in this case can be formalized as Linear Matrix Inequalities [27], which are supported by MATLAB and other software tools.

First of all, we show that any generalized homogeneous system is diffeomorphic to a standard homogeneous one. Next, a change of coordinate, which transforms asymptotically stable generalized homogeneous system to a quadratically stable one, is presented. In both cases the so-called canonical homogeneous norm [28] is utilized for construction of the corresponding coordinate transformation. Combination of these two results allows the necessary and sufficient stability condition to be presented in terms of existence of the quadratic-like Lyapunov function $V(x)=x^{\top} \Xi(x) P \Xi(x) x$, where $P=P^{\top}>0$ and the nonsingular matrix $\Xi$ is constant along any ray from the origin (i.e. $\Xi\left(e^{s} x\right)=\Xi(x)$ for $x \neq 0$, $s \in \mathbb{R})$ and $\frac{\partial \Xi(x)}{\partial x_{i}} x=0$ for $x \neq 0$. The properties of matrix $\Xi$ motivate the conjecture about existence of the quadratic Lyapunov function $(\Xi=$ const), which fails in the general case. However, some examples show that the obtained stability condition may simplify finite-time stability/stabilizability analysis and control design in some particular cases.

Notation: $\mathbb{R}$ is the set of real numbers, $e_{i}=(0, . ., 1, . ., 0)^{\top} \in$ $\mathbb{R}^{n} ; \frac{\partial}{\partial u}=\left(\frac{\partial}{\partial u_{1}}, \frac{\partial}{\partial u_{2}}, \ldots, \frac{\partial}{\partial u_{n}}\right) ; C^{n}(X, Y)$ is the set of continuously differentiable (at least up to the order $n$ ) maps $X \rightarrow Y$, where $X, Y$ are open subsets of finite dimensional spaces; $I_{n} \in \mathbb{R}^{n \times n}$ - the identity matrix; $\mathbf{0}$ denotes zero element, e.g. $\mathbf{0} \in \mathbb{R}^{n}$ is the zero vector but $\mathbf{0} \in \mathbb{R}^{n \times n}$ is the zero matrix; $\operatorname{diag}\left\{\lambda_{1}, . ., \lambda_{n}\right\}$ - diagonal matrix.

\section{Generalized Homogeneity}

Homogeneity is a sort of symmetry of an operator (function/vector field) with respect to a group of transformations that is usually called as group of dilations (or simply $\mathrm{di}$ lation). The generalized homogeneity studied in [18] deals with the groups of linear transformations (linear dilations).

\section{A. Dilation Group}

Let $\|\cdot\|$ be a norm in $\mathbb{R}^{n}$ and $\|\cdot\|_{\mathbb{A}}$ be the matrix norm induced by $\|\cdot\|$, i.e. $\|A\|_{\mathbb{A}}=\sup _{u \in \mathbb{R}^{n}} \frac{\|A u\|}{\|u\|}$ if $A \in \mathbb{R}^{n \times n}$.

Definition 1 ([18]): A map d : $\mathbb{R} \rightarrow \mathbb{R}^{n \times n}$ is called dilation in $\mathbb{R}^{n}$ if it satisfies

- Group property. $\boldsymbol{d}(0)=I_{n}, \boldsymbol{d}(t+s)=\boldsymbol{d}(t) \boldsymbol{d}(s), t, s \in \mathbb{R}$;

- Continuity property. $d$ is continuous, i.e. $\forall t>0, \forall \varepsilon>0, \exists \delta>0:|s-t|<\delta \Rightarrow\|\boldsymbol{d}(s)-\boldsymbol{d}(t)\|_{\mathbb{A}} \leq \varepsilon ;$

- Limit property. $\lim _{s \rightarrow-\infty}\|\boldsymbol{d}(s) u\|=0$ and $\lim _{s \rightarrow+\infty}\|\boldsymbol{d}(s) u\|=+\infty$ uniformly on the unit sphere $S:=\left\{u \in \mathbb{R}^{n}:\|u\|=1\right\}$. 
Obviously, the dilation $\mathbf{d}$ is a uniformly continuous group of invertible linear maps $\mathbf{d}(s)$ such that $\mathbf{d}(-s)=[\mathbf{d}(s)]^{-1}$.

The matrix $G_{\mathbf{d}} \in \mathbb{R}^{n \times n}$ defined as $G_{\mathbf{d}}=\lim _{s \rightarrow 0} \frac{\mathbf{d}(s)-I_{n}}{s}$ is known (see, e.g. [29, Ch. 1]) as the generator of the group $\mathbf{d}(s)$. It satisfies the following properties

$$
\frac{d}{d s} \mathbf{d}(s)=G_{\mathbf{d}} \mathbf{d}(s)=\mathbf{d}(s) G_{\mathbf{d}} \quad \text { and } \quad \mathbf{d}(s)=e^{G_{\mathbf{d}} s}:=\sum_{i=0}^{+\infty} \frac{s^{i} G_{\mathbf{d}}^{i}}{i !} \text {. }
$$

Denote also $\lfloor A\rfloor_{\mathbb{A}}=\inf _{u \in \mathbb{R}^{n}} \frac{\|A u\|}{\|u\|}$. Limit property implies

$\bullet\|\mathbf{d}(s)\|_{\mathbb{A}} \rightarrow \quad 0 \quad$ as $s \rightarrow-\infty ; \quad \bullet \mathbf{d}(s) \neq I_{n}$ if $s \neq 0$;

$\bullet\lfloor\mathbf{d}(s)\rfloor_{\mathbb{A}} \rightarrow+\infty$ as $s \rightarrow+\infty ; \quad \bullet\left\lfloor G_{\mathbf{d}}\right\rfloor>0\left(\operatorname{ker} G_{\mathbf{d}}=\{\mathbf{0}\}\right)$.

The most popular dilations [12], [13], [26], [15]

- uniform (or standard) dilation (L. Euler 17th century): $\mathbf{d}(s)=e^{s}, s \in \mathbb{R}$

- weighted dilation (Zubov 1958, [2]): $\mathbf{d}(s)=\left(\begin{array}{cccc}e^{r_{1} s} & 0 & \ldots & 0 \\ 0 & e^{r_{2}} s & \ldots & 0 \\ \ldots & \ldots & \ldots & \ldots \\ 0 & 0 & \ldots & e^{r_{n}} s\end{array}\right), s \in \mathbb{R}$ and $r_{i}>0, i=1, \ldots, n ;$ satisfy Definition 1 with $G_{\mathbf{d}}=I_{n}$ and $G_{\mathbf{d}}=\operatorname{diag}\left\{r_{i}\right\}$, resp.

Definition 2: Dilation d is monotone if $\|\boldsymbol{d}(s)\|_{\mathbb{A}}<1, \forall s<0$.

Monotonicity of dilation depends on the norm $\|\cdot\|$. For example, the dilation $\mathbf{d}(s)=e^{s}\left(\begin{array}{cc}\cos (s) & \sin (s) \\ -\sin (s) & \cos (s)\end{array}\right)$ with $G_{\mathbf{d}}=$ $\left(\begin{array}{cc}1 & 1 \\ -1 & 1\end{array}\right)$ is monotone on $\mathbb{R}^{2}$ equipped with weighted norm $\|u\|_{P}=\sqrt{u^{\top} P u}$ if $P=\left(\begin{array}{cc}1 & -1 / \sqrt{2} \\ -11 / \sqrt{2} & 1\end{array}\right)>0$ and it is not monotone if, for example, $P=\left(\begin{array}{cc}1 & 3 / 4 \\ 3 / 4 & 1\end{array}\right)>0$. In the latter case, the curve $\{\mathbf{d}(s) u: s \in \mathbb{R}\}$ may cross the unit sphere $S$ in two different points.

Theorem 1: The next four conditions are equivalent

1) dilation $\boldsymbol{d}$ is monotone;

2) $\lfloor\boldsymbol{d}(s)\rfloor_{\mathbb{A}}>1$ for $s>0$;

3) continuous function $\|\boldsymbol{d}(\cdot) u\|: \mathbb{R} \rightarrow \mathbb{R}_{+}$is strictly increasing for any $u \in S$;

4) for any $u \in \mathbb{R}^{n} \backslash\{\boldsymbol{0}\}$ there exists a unique pair $\left(s_{0}, u_{0}\right) \in \mathbb{R} \times S$ such that $u_{0}=\boldsymbol{d}\left(s_{0}\right) u$.

Proof. 1) $\Rightarrow$ 2) For any $u \in S$ we have $1=$ $\|u\|=\|\mathbf{d}(s) \mathbf{d}(-s) u\| \leq\|\mathbf{d}(s)\|_{\mathbb{A}}\|\mathbf{d}(-s) u\|$. Hence, $1 \leq$ $\|\mathbf{d}(s)\|_{\mathbb{A}}\lfloor\mathbf{d}(-s)\rfloor_{\mathbb{A}}$ for any $s \in \mathbb{R}$. 2) $\left.\Rightarrow \mathbf{3}\right)$ If $u \neq 0$ and $s_{1}<s_{2}$ then $\left\|\mathbf{d}\left(s_{1}\right) u\right\|-\left\|\mathbf{d}\left(s_{2}\right) u\right\|=\left\|\mathbf{d}\left(s_{1}\right) u\right\|-\| \mathbf{d}\left(s_{2}-\right.$ $\left.s_{1}\right) \mathbf{d}\left(s_{1}\right) u\left\|\leq\left(1-\left\lfloor\mathbf{d}\left(s_{2}-s_{1}\right)\right\rfloor_{\mathbb{A}}\right)\right\| \mathbf{d}\left(s_{1}\right) u \|<0$. The implication 3) $\Rightarrow$ 4) is straightforward. Indeed, since $\|\mathbf{d}(0) u\|=$ $\|u\|$ and $\|\mathbf{d}(\cdot) u\|$ is a continuous strictly increasing function, $\|\mathbf{d}(-\infty) u\|=0,\|\mathbf{d}(+\infty) u\|=+\infty$, then there exists a unique $s_{0} \in \mathbb{R}:\left\|\mathbf{d}\left(s_{0}\right) u\right\|=1$ (i.e. $u_{0}=\mathbf{d}\left(s_{0}\right) u \in S$ ). 4) $\Rightarrow$ 1) If $u \in S$ then $\mathbf{d}(s) u \notin S$ for all $s \neq 0$. Indeed, otherwise the pair $\left(s_{0}, u_{0}\right) \in \mathbb{R} \times S$ such that $u_{0}=\mathbf{d}\left(s_{0}\right) u \in$ $S$ is not unique. Hence, the limit property of the dilation implies $\|\mathbf{d}(s) u\|<1$ for all $s<0$ and all $u \in S$.

Theorem 1 also implies that the functions $\|\mathbf{d}(\cdot)\|_{\mathbb{A}}: \mathbb{R} \rightarrow \mathbb{R}_{+}$ and $\lfloor\mathbf{d}(\cdot)\rfloor_{\mathbb{A}}: \mathbb{R} \rightarrow \mathbb{R}_{+}$are continuous and strictly increasing.

Definition 3: A dilation $\boldsymbol{d}$ is said to be strictly monotone on $\mathbb{R}^{n}$ if $\exists \beta>0$ such that $\|\boldsymbol{d}(s)\|_{\mathbb{A}} \leq e^{\beta s}$ for $s \leq 0$.

The dilation $\mathbf{d}$ considered in the above example is strictly monotone on $\mathbb{R}^{2}$ equipped with the Euclidean norm.

Theorem 2: Let d be a dilation in $\mathbb{R}^{n}$ then

- the matrix $-G_{\boldsymbol{d}}$ is Hurwitz, i.e. all eigenvalues $\lambda_{i}$ of $G_{\boldsymbol{d}}$ have positive real parts, $\beta^{*}=\min \Re\left(\lambda_{i}\right)>0$;
- for any $\beta \in\left(0, \beta^{*}\right]$ there exists a matrix $P \in \mathbb{R}^{n \times n}$ :

$$
P G_{\boldsymbol{d}}+G_{\boldsymbol{d}}^{\top} P \geq 2 \beta P, \quad P=P^{\top}>0,
$$

- the dilation $\boldsymbol{d}$ is strictly monotone with respect to the weighted Euclidean norm $\|\cdot\|=\sqrt{\langle\cdot, \cdot\rangle}$ induced by the inner product $\langle u, v\rangle=u^{\top} P v$ with $P$ satisfying (1): $e^{\alpha s} \leq\lfloor\boldsymbol{d}(s)\rfloor_{\mathbb{A}} \leq\|\boldsymbol{d}(s)\|_{\mathbb{A}} \leq e^{\beta s}$ for $s \leq 0$ and $e^{\beta s} \leq$ $\lfloor\boldsymbol{d}(s)\rfloor_{\mathbb{A}} \leq\|\boldsymbol{d}(s)\|_{\mathbb{A}} \leq e^{\alpha s}$ for $s \geq 0$, where $\alpha=\frac{\lambda_{\max }(Q)}{2}$, $\beta=\frac{\lambda_{\min }(Q)}{2}, Q=P^{\frac{1}{2}} G_{\boldsymbol{d}} P^{-\frac{1}{2}}+P^{-\frac{1}{2}} G_{\boldsymbol{d}}^{\top} P^{\frac{1}{2}}$.

Proof. Since $\frac{d}{d s} \mathbf{d}(s)=G_{\mathbf{d}} \mathbf{d}(s), \mathbf{d}(0)=I$ then $\mathbf{d}(s)$ is the fundamental matrix of the linear system ODEs with the matrix $G_{\mathbf{d}}$. The limit property of the dilation implies that this system of ODEs is globally asymptotically stable in the inverse time, i.e. the matrix $-G_{\mathbf{d}}$ is Hurwitz. Hence, there exists a symmetric positive definite matrix such that (1) holds and for any $u \in S$ one has $\frac{d}{d s}\|\mathbf{d}(s) u\|^{2}=$ $u^{\top} \mathbf{d}(s)^{\top}\left(G_{\mathbf{d}}^{\top} P+P G_{\mathbf{d}}\right) \mathbf{d}(s) u \geq 2 \beta\|\mathbf{d}(s) u\|^{2}$. Similarly we derive $\frac{d}{d s}\|\mathbf{d}(s) u\|^{2} \leq 2 \alpha\|\mathbf{d}(s) u\|^{2}$, i.e. the inequalities for matrix norms also hold.

Therefore, any dilation $\mathbf{d}$ is strictly monotone on $\mathbb{R}^{n}$ equipped with the weighted Euclidian norm $\|u\|=\sqrt{u^{\top} P u}$ provided that the matrix $P>0$ satisfies (1) for some $\beta>0$.

\section{B. Canonical Homogeneous Norm}

Dilation d introduces a new topology in $\mathbb{R}^{n}$ (spheres and balls) by means of the "homogeneous norm" [4], [14], [13].

Definition 4: A continuous function $p: \mathbb{R}^{n} \rightarrow \mathbb{R}_{+}$is said to be $\boldsymbol{d}$-homogeneous norm if $p(u) \rightarrow 0$ as $u \rightarrow \mathbf{0}$ and $p(\boldsymbol{d}(s) u)=e^{s} p(u)>0$ for $u \in \mathbb{R}^{n} \backslash\{\mathbf{0}\}$ and $s \in \mathbb{R}$.

For monotone dilations the canonical homogeneous norm $\|\cdot\|_{\mathbf{d}}: \mathbb{R}^{n} \rightarrow \mathbb{R}_{+}$is defined as follows:

$\|u\|_{\mathbf{d}}=e^{s_{u}}$ where $s_{u} \in \mathbb{R}$ such that $\left\|\mathbf{d}\left(-s_{u}\right) u\right\|=1$.

In [28] such a homogeneous norm was called canonical since it is induced by the canonical norm $\|\cdot\|$ in $\mathbb{R}^{n}$ and $\|x\|_{\mathbf{d}}=\|x\|=1$ on the unit sphere $S$. Obviously, $\left\lfloor\mathbf{d}\left(\ln \|u\|_{\mathbf{d}}\right)\right\rfloor_{\mathbb{A}} \leq\|u\| \leq\left\|\mathbf{d}\left(\ln \|u\|_{\mathbf{d}}\right)\right\|_{\mathbb{A}}$.

Note that $\|\cdot\|_{\mathbf{d}}=\|\cdot\|$ if $\mathbf{d}(s)=e^{s} I_{n}$ - uniform dilation.

Proposition 1: If $\mathbf{d}$ is a strictly monotone dilation then

- $\left|\left\|u_{1}\right\|_{\mathbf{d}}^{\beta}-\left\|u_{2}\right\|_{\mathbf{d}}^{\beta}\right| \leq\left\|u_{1}-u_{2}\right\|$ for $u_{1}, u_{2} \in \mathbb{R}^{n} \backslash B_{\mathbf{d}}(1)$,

- $\|\cdot\|_{\mathbf{d}}$ is Lipschitz continuous outside the origin;

- if the norm $\|\cdot\|$ is smooth outside the origin then the homogeneous norm $\|\cdot\|_{\mathbf{d}}$ is also smooth outside the origin, $\frac{d\|\mathbf{d}(-s) u\|}{d s}<0$ if $s \in \mathbb{R}, u \in \mathbb{R}^{n} \backslash\{\mathbf{0}\}$ and

$$
\frac{\partial\|u\|_{\mathbf{d}}}{\partial u}=\frac{\left.\|u\|_{\mathbf{d}} \frac{\partial\|z\|}{\partial z}\right|_{z=\mathbf{d}(-s) u}}{\left.\frac{\partial\|z\|}{\partial z}\right|_{z=\mathbf{d}(-s) u} G_{\mathbf{d}} \mathbf{d}(-s) u} \mid
$$

Proof. Since for $u_{i} \in \mathbb{R}^{n}$ we have $\left\|u_{i}\right\|_{\mathbf{d}}=e^{s_{i}}$ : $\left\|\mathbf{d}\left(-s_{i}\right) u\right\|=1$ then $1=\left\|\mathbf{d}\left(-s_{1}\right) u_{1}\right\|=\| \mathbf{d}\left(-s_{1}\right)\left(u_{1}-\right.$ $\left.u_{2}\right)+\mathbf{d}\left(s_{2}-s_{1}\right) \mathbf{d}\left(-s_{2}\right) u_{2}\|\leq\| \mathbf{d}\left(-s_{1}\right)\left\|_{\mathbb{A}}\right\|\left(u_{1}-u_{2}\right) \|+$ $\left\|\mathbf{d}\left(s_{2}-s_{1}\right)\right\|_{\mathbb{A}}$. For $1<\left\|u_{2}\right\|_{\mathbf{d}}<\left\|u_{1}\right\|_{\mathbf{d}}$ we have $0<s_{2}<$ $s_{1}$ and $1 \leq e^{-\beta s_{1}}\left\|u_{1}-u_{2}\right\|+e^{\beta s_{2}-\beta s_{1}}$ or equivalently, $\left\|u_{1}\right\|_{\mathbf{d}}-\left\|u_{2}\right\|_{\mathbf{d}} \leq\left\|u_{1}-u_{2}\right\|$. Lipschitz continuity follows from the proven inequality, the identity $\|\mathbf{d}(s) u\|_{\mathbf{d}}=e^{s}\|u\|_{\mathbf{d}}$ and monotonicity of the dilation. The existence of the unique function $s: \mathbb{R}^{n} \rightarrow \mathbb{R}$ such that $\|\mathbf{d}(-s(u)) u\|=1$ has been proven in Theorem 1. Since the dilation is strictly monotone then $\frac{d}{d s}\|\mathbf{d}(-s) u\|<0$ on $S$ (and, on $\mathbb{R}^{n} \backslash\{0\}$ ) 
for all $s \in \mathbb{R}$ (see, Theorem 1). Since the norm $\|\cdot\|$ is smooth them $\frac{d}{d s}\|\mathbf{d}(-s) u\|=-\left.\frac{\partial\|z\|}{\partial z}\right|_{z=\mathbf{d}(-s) u} G_{\mathbf{d}} \mathbf{d}(-s) u$. Taking into account $\frac{\partial}{\partial u}\|u\|_{\mathbf{d}}=\left.e^{s} \frac{\partial s}{\partial u}\right|_{s=\ln \|u\|_{\mathbf{d}}}$ the formula (3) can be derived using Implicit Function Theorem [30] applied to the equation $\|\mathbf{d}(-s) u\|=1$ (see, also [28]).

\section{Generalized Homogeneous Functions and Vectors Fields}

Homogeneous vector field have a lot of properties useful for control design and state estimation as well as for analysis of convergence rates [5], [23], [31], [26].

Definition 5: A vector field $f: \mathbb{R}^{n} \rightarrow \mathbb{R}^{n}$ (a function $\left.h: \mathbb{R}^{n} \rightarrow \mathbb{R}\right)$ is said to be d-homogeneous of degree $\nu \in \mathbb{R}$ if

$$
f(\mathbf{d}(s) u)=e^{\nu s} \mathbf{d}(s) f(u), \quad \forall u \in \mathbb{R}^{n} \backslash\{\mathbf{0}\}, \quad \forall s \in \mathbb{R} .
$$

(resp. $\left.h(\mathbf{d}(s) u)=e^{\nu s} h(u), \quad \forall u \in \mathbb{R}^{n} \backslash\{\mathbf{0}\}, \quad \forall s \in \mathbb{R}.\right)$ Example 1: Let us consider the dilation $\mathbf{d}(s)=$ $e^{s}\left(\begin{array}{ccc}1 & 0 & 0 \\ 0 & \cos (s) & \sin ^{2}(s) \\ 0 & -\sin (s) & \cos (s)\end{array}\right)$ that is strictly monotone with respect to the Euclidean norm $\|x\|=\sqrt{x^{T} x}$ and $G_{\mathbf{d}}=\left(\begin{array}{ccc}1 & 0 & 0 \\ 0 & 1 & 1 \\ 0 & -1 & 1\end{array}\right)$. The vector field $f: \mathbb{R}^{3} \rightarrow \mathbb{R}^{3}$ defined as $f(x)=$ $\left(\begin{array}{c}x_{2}^{2}+x_{3}^{2} \\ x_{1}^{2}\left(\cos \left(\ln \left|x_{1}\right|\right)+\sin \left(\ln \left|x_{1}\right|\right)\right) \\ x_{1}^{2}\left(\cos \left(\ln \left|x_{1}\right|\right)-\sin \left(\ln \left|x_{1}\right|\right)\right)\end{array}\right)$ and the function $h: \mathbb{R}^{3} \rightarrow \mathbb{R}$ given by $h=x_{1}^{3}+\left(x_{2}^{2}+x_{3}^{2}\right)^{\frac{3}{2}}$ are $\mathbf{d}$-homogeneous of degree 1 and 3 , respectively.

Example 2 (Homogeneous vector field of any degree):

The vector field may have different degrees of homogeneity dependently of the dilation group. Indeed, the linear vector field $f: \mathbb{R}^{n} \rightarrow \mathbb{R}^{n}, f(x)=A x$ defined by a chain of integrators $A=\left(\begin{array}{cc}0 & I_{n-1} \\ 0 & 0\end{array}\right) \in \mathbb{R}^{n \times n}$ is $\mathbf{d}_{1}$-homogeneous of degree $\mu \in[0,1]$ with $\mathbf{d}_{1}(s)=\operatorname{diag}\left\{e^{(n+(i-1) \mu) s}\right\}_{i=1}^{n}$ and $\mathbf{d}_{2}$-homogeneous of degree $\mu \in[-1,0]$ with $\mathbf{d}_{2}(s)=\operatorname{diag}\left\{e^{(n+(n-i) \mu) s}\right\}_{i=1}^{n}$.

Let $\mathbb{F}_{\mathbf{d}}\left(\mathbb{R}^{n}\right) \quad$ (resp. $\mathbb{H}_{\mathbf{d}}\left(\mathbb{R}^{n}\right)$ ) be the set of $\mathbf{d}$ homogeneous vector fields $\mathbb{R}^{n} \rightarrow \mathbb{R}^{n}$ (resp. functions $\left.\mathbb{R}^{n} \rightarrow \mathbb{R}\right)$, which are continuous on $\mathbb{R}^{n} \backslash\{\mathbf{0}\}$. Let $\operatorname{deg}_{\mathbf{d}}(h)$ (resp. $\left.\operatorname{deg}_{\mathbf{d}}(f)\right)$ denote the homogeneity degree of $h \in$ $\mathbb{H}_{d}\left(\mathbb{R}^{n}\right)$ (resp. $f \in \mathbb{F}_{\mathbf{d}}\left(\mathbb{R}^{n}\right)$ ).

The homogeneity allows local properties (e.g. smoothness) of vector fields (functions) to be extended globally [2], [3].

Corollary 1: The vector field $f \in \mathbb{F}_{\boldsymbol{d}}\left(\mathbb{R}^{n}\right)$ is Lipschitz. continuous (smooth) on $\mathbb{R}^{n} \backslash\{\mathbf{0}\}$ if and only if it satisfies Lipschitz condition (it is smooth) on $S$ provided that $\boldsymbol{d}$ is strictly monotone on $\mathbb{R}^{n}$ equipped with a (smooth) norm $\|\cdot\|$. Proof. The necessity is straightforward since any locally Lipschitz function on a compact set satisfies Lipschitz condition on it. Let us prove sufficiency. Let $u_{i} \in \mathbb{R}^{n} \backslash\{\mathbf{0}\}, i=1,2$ then $u_{i}=\mathbf{d}\left(\ln \left\|u_{i}\right\|_{\mathbf{d}}\right) z_{i}$ for some $z_{i} \in S, f\left(u_{1}\right)-f\left(u_{2}\right)=$ $f\left(\mathbf{d}\left(\ln \left\|u_{1}\right\|_{\mathbf{d}}\right) z_{1}\right)-f\left(\mathbf{d}\left(\ln \left\|u_{2}\right\|_{\mathbf{d}}\right) z_{2}\right)=\left\|u_{1}\right\|_{\mathbf{d}}^{\nu} \mathbf{d}\left(\ln \left\|u_{1}\right\|_{\mathbf{d}}\right)$. $f\left(z_{1}\right)-\left\|u_{2}\right\|_{\mathbf{d}}^{\nu} \mathbf{d}\left(\ln \left\|u_{2}\right\|_{\mathbf{d}}\right) f\left(z_{2}\right)=\left\|u_{1}\right\|_{\mathbf{d}}^{\nu} \mathbf{d}\left(\ln \left\|u_{1}\right\|_{\mathbf{d}}\right)\left(f\left(z_{1}\right)-\right.$ $\left.f\left(z_{2}\right)\right)+\left(\left\|u_{1}\right\|_{\mathbf{d}}^{\nu} \mathbf{d}\left(\ln \left\|u_{1}\right\|_{\mathbf{d}}\right)-\left\|u_{2}\right\|^{\nu} \mathbf{d}\left(\ln \left\|u_{1}\right\|_{\mathbf{d}}\right) f\left(z_{2}\right)+\right.$ $\left\|u_{2}\right\|^{\nu}\left(\mathbf{d}\left(\ln \left\|u_{1}\right\|_{\mathbf{d}}\right)-\mathbf{d}\left(\ln \left\|u_{2}\right\|_{\mathbf{d}}\right)\right) f\left(z_{2}\right)$. If $L>0$ is a Lipschitz constant on $S$ then $\left\|f\left(u_{1}\right)-f\left(u_{2}\right)\right\| \leq$ $L\left\|u_{1}\right\|_{\mathbf{d}}^{\nu} \mathbf{d}\left(\ln \left\|u_{1}\right\|_{\mathbf{d}}\right)\left\|z_{1}-z_{2}\right\|+\left\|\mathbf{d}\left(\ln \left\|u_{1}\right\|_{\mathbf{d}}\right) f\left(z_{2}\right)\right\|\left(\left\|u_{1}\right\|_{\mathbf{d}}^{\nu}-\right.$ $\left.\left\|u_{2}\right\|_{\mathbf{d}}^{\nu}\right)+\left\|f\left(z_{2}\right)\right\|\left\|u_{2}\right\|^{\nu}\left\|\mathbf{d}\left(\ln \left\|u_{1}\right\|_{\mathbf{d}}\right)-\mathbf{d}\left(\ln \left\|u_{2}\right\|_{\mathbf{d}}\right)\right\|_{\mathbb{A}}$. Since
$\mathbf{d}\left(s_{1}\right)-\mathbf{d}\left(s_{2}\right)=G_{\mathbf{d}} \int_{s_{2}}^{s_{1}} \mathbf{d}(s) d s$ and the function $\|\mathbf{d}(\cdot)\|_{\mathbb{A}}$ is strictly monotone increasing then $\| \mathbf{d}\left(\ln \left\|u_{1}\right\|_{\mathbf{d}}\right)-$ $\left.\mathbf{d}\left(\ln \left\|u_{2}\right\|_{\mathbf{d}}\right)\right)\left\|_{\mathbb{A}} \leq M\right\| G_{\mathbf{d}}\left\|\left|\ln \left\|u_{1}\right\|_{\mathbf{d}}-\ln \left\|u_{2}\right\|_{\mathbf{d}}\right|\right.$, where $M=\max \left\{\left\|\mathbf{d}\left(\ln \left\|u_{1}\right\|_{\mathbf{d}}\right)\right\|_{\mathbb{A}},\left\|\mathbf{d}\left(\ln \left\|u_{2}\right\|_{\mathbf{d}}\right)\right\|_{\mathbb{A}}\right\}$. Since the homogeneous norm is Lipschitz continuous on $\mathbb{R}^{n} \backslash\{\mathbf{0}\}$ but power and logarithm functions are Lipschitz continuous outside zero then $f$ is Lipschitz continuous outside the origin. (Differentiability of homogeneous vector field (function) $f$ on $\mathbb{R}^{n} \backslash\{\mathbf{0}\}$ can be proven by means of the formula (3), the identity $\frac{d}{d s} \mathbf{d}(s)=G_{\mathbf{d}} \mathbf{d}(s)$ and the representation $f(u)=$ $\|u\|_{\mathbf{d}}^{\nu} \mathbf{d}\left(\ln \|u\|_{\mathbf{d}}\right) f(z)$ with $z=\mathbf{d}(-\ln \|u\|) u \in S$.)

If a function (or a vector field) is smooth then homogeneity is inherited by its derivatives in a certain way.

Corollary 2: If $h \in \mathbb{H}_{\boldsymbol{d}}\left(\mathbb{R}^{n}\right) \cap C^{1}\left(\mathbb{R}^{n} \backslash\{\mathbf{0}\}, \mathbb{R}\right)$ then

$$
\begin{gathered}
e^{\operatorname{deg}(h) s \frac{\partial h(u)}{\partial u}}=\left.\frac{\partial h(z)}{\partial z}\right|_{z=\boldsymbol{d}(s) u} \boldsymbol{d}(s), \\
\frac{\partial h(u)}{\partial u} G_{\boldsymbol{d}} u=\operatorname{deg}_{\boldsymbol{d}}(h) h(u),
\end{gathered}
$$

for $u \in \mathbb{R}^{n} \backslash\{\mathbf{0}\}$ and $s \in \mathbb{R}$.

Proof. The formula (5) can be obtained using the definition of the (Frechét) derivative, which coincides with $\frac{\partial h}{\partial u}$ if $h$ is smooth. Namely, $\lim _{\|\Delta\| \rightarrow 0} \frac{\left|h(u+\Delta)-h(u)-\frac{\partial h(u)}{\partial u} \Delta\right|}{\|\Delta\|}=0$ and $\lim _{\|\Delta\| \rightarrow 0} \frac{\left|h(\mathbf{d}(s) u+\Delta)-h(\mathbf{d}(s) u)-\frac{\partial h(z)}{\partial z}\right|_{z=\mathbf{d}(s) u} \Delta \mid}{\|\Delta\|}=0$ where $\Delta \in \mathbb{R}^{n}$. Since $h \in \mathbb{H}_{\mathbf{d}}$ then $\frac{\left|h(\mathbf{d}(s) u+\Delta)-h(\mathbf{d}(s) u)-\frac{\partial h(z)}{\partial z}\right|_{z=\mathbf{d}(s) u} \Delta \mid}{\|\Delta\|}=$ $e^{\nu s} \frac{\left|h(u+\tilde{\Delta})-h(u)-e^{-\nu s} \frac{\partial h(z)}{\partial z}\right|_{z=\mathbf{d}(s) u} \mathbf{d}(s) \tilde{\Delta} \mid}{\|\mathbf{d}(s) \tilde{\Delta}\|} \leq \frac{e^{\nu s}}{[\mathbf{d}(s)\rfloor_{\mathbb{A}}} \times$ $\frac{\left|h(u+\tilde{\Delta})-h(u)-e^{-\nu s} \frac{\partial h(z)}{\partial z}\right|_{z=\mathbf{d}(s) u} \mathbf{d}(s) \tilde{\Delta} \mid}{\|\tilde{\Delta}\|}$, where $\nu=\operatorname{deg}(h)$ and $\tilde{\Delta}=\mathbf{d}(-s) \Delta$ such that $\|\tilde{\Delta}\| \rightarrow 0$ implies $\|\Delta\| \rightarrow 0$. Therefore the identity (5) holds.

To prove (6) let us consider a homogeneous norm $\|\cdot\|_{\mathbf{d}}$ induced by a weighted Euclidean norm $\|u\|=\sqrt{u^{\top} P u}$ in $u \in \mathbb{R}^{n} \backslash\{\mathbf{0}\}, P=P^{\top}>0$. In this case, due to (3) we have $\frac{\partial\|u\|_{\mathbf{d}}}{\partial u} u>0$ if $u \neq 0$. From $h(u)=\|u\|_{\mathbf{d}}^{\nu} h\left(\mathbf{d}\left(-\ln \|u\|_{\mathbf{d}}\right) u\right)$ we derive $\frac{\partial h(u)}{\partial u}=\nu h\left(\mathbf{d}\left(-\ln \|u\|_{\mathbf{d}}\right) u\right)\|u\|_{\mathbf{d}}^{\nu-1} \frac{\partial\|u\|_{\mathbf{d}}}{\partial u}+$ $\|u\|_{\mathbf{d}}^{\nu} \frac{\partial h\left(\mathbf{d}\left(-\ln \|u\|_{\mathbf{d}} u\right)\right)}{\partial u}=\nu h\left(\mathbf{d}\left(-\ln \|u\|_{\mathbf{d}}\right) u\right)\|u\|_{\mathbf{d}}^{\nu-1} \frac{\partial\|u\|_{\mathbf{d}}}{\partial u}+$ $\left.\|u\|_{\mathbf{d}}^{\nu} \frac{\partial h(z)}{\partial z}\right|_{z=\mathbf{d}\left(-\ln \|u\|_{\mathbf{d}}\right) u} ^{\partial u} \frac{\partial}{\partial u}\left(\mathbf{d}\left(-\ln \|u\|_{\mathbf{d}}\right) u\right)=$ $\nu h\left(\mathbf{d}\left(-\ln \|u\|_{\mathbf{d}}\right) u\right)\|u\|_{\mathbf{d}}^{\nu-1} \frac{\partial\|u\|_{\mathbf{d}}}{\partial u}+\|u\|_{\mathbf{d}}^{\nu} \frac{\partial h(z)}{\partial z}$ $\times\left(\mathbf{d}\left(-\ln \|u\|_{\mathbf{d}}\right)-\frac{G_{\mathbf{d}} \mathbf{d}\left(-\ln \|u\|_{\mathbf{d}}\right) u}{\|u\|_{\mathbf{d}}} \frac{\partial\|u\|_{\mathbf{d}}}{\partial u}\right) . \quad \begin{aligned} & \text { Therefore, for } \\ & \text { (n) }\end{aligned}$ $\|u\|=1$ we have $\frac{\partial h(u)}{\partial u} G_{\mathbf{d}} u \frac{\partial\|u\|_{\mathbf{d}}}{\partial u}=\nu h(u) \frac{\partial\|u\|_{\mathbf{d}}}{\partial u}$. Hence, multiplying by $u$ we obtain that (6) holds for $\|u\|=1$. Since $\|\cdot\|_{n e w}=\gamma\|\cdot\|$ with $\gamma>0$ is again a weighted Euclidean norm in $\mathbb{R}^{n}$ then the obtained identity holds on $\mathbb{R}^{n} \backslash\{\mathbf{0}\}$.

Remark 1: If $\boldsymbol{d}$ is a dilation with the generator $G_{\boldsymbol{d}}$ then for any $\alpha>0$ the group $\boldsymbol{d}^{\alpha}$ defined as $\boldsymbol{d}^{\alpha}(s):=\boldsymbol{d}(\alpha s), s \in \mathbb{R}^{n}$ is the dilation with the generator $G_{\boldsymbol{d}^{\alpha}}=\alpha G_{\boldsymbol{d}}$. Moreover, if $f \in \mathbb{F}_{\boldsymbol{d}}\left(\mathbb{R}^{n}\right)$ then $f \in \mathbb{F}_{\boldsymbol{d}^{\alpha}}\left(\mathbb{R}^{n}\right)$ and $\operatorname{deg}_{\boldsymbol{d}^{\alpha}}(f)=\alpha \operatorname{deg}_{\boldsymbol{d}}(f)$.

\section{Stability of HomogeneOUS Systems}

Homogeneity may simplify an analysis of differential equations, e.g. to prove existence and uniqueness of solution on $\mathbb{R}^{n} \backslash\{\mathbf{0}\}$ it is sufficient to prove that the right hand side is Lipschitz continuous (or differentiable) on a sphere 
The most important property of $\mathbf{d}$-homogeneous systems is scalability of the solutions [2], [4], [12], [31], [32], [18].

Theorem 3: If $\varphi_{\xi_{0}}:[0, T) \rightarrow \mathbb{R}^{n}$ is a solution to

$$
\dot{\xi}=f(\xi), \quad f \in \mathbb{F}_{\boldsymbol{d}}\left(\mathbb{R}^{n}\right)
$$

with the initial condition $\xi(0)=\xi_{0} \in \mathbb{R}^{n}$ then $\varphi_{\boldsymbol{d}(s) \xi_{0}}$ : $\left[0, e^{-\nu s} T\right) \rightarrow \mathbb{R}^{n}$ defined as $\varphi_{\boldsymbol{d}(s) \xi_{0}}(t):=\boldsymbol{d}(s) \varphi_{\xi_{0}}\left(t e^{\nu s}\right)$ with $s \in \mathbb{R}$ is a solution to (7) with the initial condition $\xi(0)=\boldsymbol{d}(s) \xi_{0}$, where $\nu=\operatorname{deg}_{\boldsymbol{d}}(f)$.

Proof. The scheme of the proof is standard (see [2]) for any type of homogeneity. Since $\frac{d \varphi_{\xi_{0}}(t)}{d t}=f\left(\varphi_{\xi_{0}}(t)\right)$ then $\mathbf{d}(s) \frac{d \varphi_{\xi_{0}}}{d t}=\frac{d \mathbf{d}(s) \varphi_{\xi_{0}}}{d t}=\mathbf{d}(s) f\left(\varphi_{\xi_{0}}\right)=e^{-\nu s} f\left(\mathbf{d}(s) \varphi_{\xi_{0}}\right)$. The change of time $t=e^{\nu s} t^{\text {new }}$ completes the proof.

Now using the classical result [2], [12] about existence of Lyapunov functions we prove equivalence of asymptotically stable homogeneous system to a quadratically stable one.

Theorem 4: The next five claims are equivalent

1) The origin of the system (7) is asymptotically stable.

2) $\exists V \in \mathbb{H}_{\boldsymbol{d}}\left(\mathbb{R}^{n}\right) \cap C^{\infty}\left(\mathbb{R}^{n}\right)$ - a Lyapunov function for (7);

3) The origin of the system

$$
\dot{z}=\|z\|^{1+\operatorname{deg}_{\boldsymbol{d}}(f)}\left(\frac{\left(I_{n}-G_{\boldsymbol{d}}\right) z^{\top} z P}{z^{\top} P G_{\boldsymbol{d}} z}+I_{n}\right) f\left(\frac{z}{\|z\|}\right)
$$

is asymptotically stable, where $\|z\|=\sqrt{z^{\top} P z}$ with

$$
P G_{\boldsymbol{d}}+G_{\boldsymbol{d}}^{\top} P>0, \quad 0<P=P^{\top} \in \mathbb{R}^{n \times n} .
$$

4) For any matrix $P \in \mathbb{R}^{n \times n}$ satisfying (9) there exists a map $\Psi \in \mathbb{F}_{\boldsymbol{d}}\left(\mathbb{R}^{n}\right) \cap C^{\infty}\left(\mathbb{R}^{n} \backslash\{\mathbf{0}\}\right), \operatorname{deg}_{\boldsymbol{d}}(\Psi)=0$ such that $\Psi$ is diffemorphism on $\mathbb{R}^{n} \backslash\{\mathbf{0}\}$, homeomorphism on $\mathbb{R}^{n}, \Psi(\xi) \rightarrow \mathbf{0}$ as $\xi \rightarrow \mathbf{0}$ and

$$
\frac{\partial\left(\Psi^{\top}(\xi) P \Psi(\xi)\right)}{\partial \xi} f(\xi)<0 \text { if } \Psi^{\top}(\xi) P \Psi(\xi)=1 \text {. }
$$

Moreover, $\|\Psi\|_{\boldsymbol{d}} \in \mathbb{H}_{\boldsymbol{d}}\left(\mathbb{R}^{n}\right) \cap C^{\infty}\left(\mathbb{R}^{n} \backslash\{\mathbf{0}\}\right)$ is Lyapunov function to the system (7), where $\|\cdot\|_{d}$ is the canonical homogeneous norm induced by $\|\xi\|=\sqrt{\xi^{\top} P \xi}$.

5) For any matrix $P \in \mathbb{R}^{n \times n}$ satisfying (9) there exists a map $\Xi \in C^{\infty}\left(\mathbb{R}^{n} \backslash\{\mathbf{0}\}, \mathbb{R}^{n \times n}\right)$ such that $\operatorname{det}(\Xi(z)) \neq 0$, $\frac{\partial \Xi(z)}{\partial z_{i}} z=0, \Xi\left(e^{s} z\right)=\Xi(z)$ for $z \in \mathbb{R}^{n} \backslash\{\mathbf{0}\}, s \in \mathbb{R}$ and

$z^{\top} \Xi^{\top}(z) P \Xi(z)\left(\frac{\left(I_{n}-G_{\boldsymbol{d}}\right) z z^{\top} P}{z^{\top} P G_{\boldsymbol{d}} z}+I_{n}\right) f\left(\frac{z}{\sqrt{z^{\top} P z}}\right)<0$.

Proof. Without loss of generality we assume that $f$ is continuous at zero and $f(\mathbf{0})=\mathbf{0}$, since asymptotic stability of (8) is equivalent to asymptotic stability of $\dot{\xi}=\tilde{f}(\xi):=$ $\|\xi\|_{\mathbf{d}}^{-\operatorname{deg}_{\mathbf{d}}(f)} f(\xi)$, which is always continuous at the origin.

1) $\Leftrightarrow 2)$ We use the scheme developed in [12]. The Converse Lyapunov Theorem (see, e.g. [33] implies that there exists a smooth Lyapunov function $V: \mathbb{R}^{n} \rightarrow \mathbb{R}_{+}$. Let the smooth function $a: \mathbb{R} \rightarrow \mathbb{R}_{+}$be defined as $a(\rho)=e^{\frac{1}{1-\rho}}$ if $\rho>1$ and $a(\rho)=0$ if $\rho \leq 1$. Obviously, $a^{\prime}(\rho)>0$ if $\rho>1$. Then the function $V_{h}: \mathbb{R}^{n} \rightarrow \mathbb{R}_{+}$defined as $V_{h}(x)=$ $\int_{-\infty}^{+\infty} e^{-s} a(V(\mathbf{d}(s) x)) d s$ is $\mathbf{d}$-homogeneous Lyapunov function to the system (7). Indeed, it is well-defined (due to cut-off function $a$ ), smooth, positive definite and radially unbounded. Finally, $V_{h}$ is d-homogeneous $V_{h}(\mathbf{d}(q) x)=$ $\int_{-\infty}^{+\infty} e^{-s} a(V(\mathbf{d}(-s+q) x)) d s=e^{q} V_{h}(x)$ and $\dot{V}_{h}(x)=$ $\left.\int_{-\infty}^{+\infty} e^{-s} a^{\prime}(V(\mathbf{d}(-s) x)) \frac{\partial V(z)}{\partial z}\right|_{z=\mathbf{d}(s) x} \mathbf{d}(s) f(x) d s<0$ since $\left.\frac{\partial V(z)}{\partial z}\right|_{z=\mathbf{d}(s) x} \mathbf{d}(s) f(x)=\left.\frac{1}{e^{\nu s}} \frac{\partial V(z)}{\partial z} f(z)\right|_{z=\mathbf{d}(s) x}<0$.

1) $\Leftrightarrow$ 3) Since $P$ satisfies (1) then the dilation $\mathbf{d}$ is strictly monotone on $\mathbb{R}^{n}$ equipped with the norm $\|\xi\|=\sqrt{\xi^{T} P \xi}$.
The change of coordinates $z=\|\xi\|_{\mathbf{d}} \mathbf{d}\left(-\ln \|\xi\|_{\mathbf{d}}\right) \xi$ gives $\|z\|=\|\xi\|_{\mathbf{d}}$ and $\xi=\mathbf{d}(\ln \|z\|) \frac{z}{\|z\|}$,

$$
\begin{array}{r}
\dot{z}=\left(I_{n}-G_{\mathbf{d}}\right) \mathbf{d}\left(-\ln \|\xi\|_{\mathbf{d}}\right) \xi \frac{d\|\xi\|_{\mathbf{d}}}{d t}+\|\xi\|_{\mathbf{d}} \mathbf{d}\left(-\ln \|\xi\|_{\mathbf{d}}\right) f(\xi)= \\
\|\xi\|_{\mathbf{d}}\left(I_{n}-G_{\mathbf{d}}\right) \mathbf{d}\left(-\ln \|\xi\|_{\mathbf{d}}\right) \xi \frac{\xi^{\top} \mathbf{d}^{\top}\left(-\ln \|\xi\|_{\mathbf{d}}\right) P \mathbf{d}\left(-\ln \|\xi\|_{\mathbf{d}}\right) f(\xi)}{\xi^{\top} \mathbf{d}^{\top}\left(-\ln \|\xi\|_{\mathbf{d}}\right) P G_{\mathbf{d}} \mathbf{d}\left(-\ln \|\xi\|_{\mathbf{d}}\right) \xi}+ \\
+\|\xi\|_{\mathbf{d}} \mathbf{d}\left(-\ln \|\xi\|_{\mathbf{d}}\right) f(\xi)= \\
\|\xi\|_{\mathbf{d}}\left(\frac{\left(I-G_{\mathbf{d}}\right) \mathbf{d}\left(-\ln \|\xi\|_{\mathbf{d}}\right) \xi \xi \xi^{\top} \mathbf{d}^{\top}\left(-\ln \|\xi\|_{\mathbf{d}}\right) P}{\xi^{\top} \mathbf{d}^{\top}\left(-\ln \|\xi\|_{\mathbf{d}}\right) P G_{\mathbf{d}} \mathbf{d}\left(-\ln \|\xi\|_{\mathbf{d}}\right) \xi}+I_{n}\right) \mathbf{d}\left(-\ln \|\xi\|_{\mathbf{d}}\right) f(\xi)
\end{array}
$$

Taking into account $f \in \mathbb{F}_{\mathbf{d}}\left(\mathbb{R}^{n}\right)$ we derive (8).

4) $\Rightarrow$ 2) Note that $\Psi(\xi) \neq \mathbf{0}$ for $\xi \neq 0$, otherwise (i.e. $\exists \xi^{*} \neq 0: \Psi\left(\xi^{*}\right)=0$ ), due to homogeneity we derive that $\Psi(\xi)=\mathbf{0}$ on a smooth curve $\left\{\mathbf{d}(s) \xi^{*}, s \in \mathbb{R}\right\}$, which starts at the origin goes to $\infty$. The latter contradicts the assumption that $\Psi$ is diffeomorphism (continuously differentiable invertible map with continuously differentiable inverse) on $\mathbb{R}^{n} \backslash\{\mathbf{0}\}$. Since $\operatorname{deg}_{\mathbf{d}}(\Psi)=0$ then $\|\Psi(\mathbf{d}(s) \xi)\|_{\mathbf{d}}=$ $\|\mathbf{d}(s) \Psi(\xi)\|_{\mathbf{d}}=e^{s}\|\Psi(\xi)\|_{\mathbf{d}}$ and the function $\|\Psi(\cdot)\|_{\mathbf{d}}$ is $\mathbf{d}-$ homogeneous of degree 1 , radially unbounded, continuous at the origin and continuously differentiable outside the origin. Due to (3) the inequality $\left.\frac{\partial\|\Psi(\xi)\|}{\partial \xi} f(\xi)\right|_{\|\Psi(\xi)\|=1}<0$ implies $\left.\frac{\partial\|\Psi(\xi)\|_{\mathbf{d}}}{\partial \xi} f(\xi)\right|_{\|\Psi(\xi)\|=1}<0$. Applying homogeneity we derive $\frac{\partial\|\Psi(\xi)\|_{\mathbf{d}}}{\partial \xi} f(\xi)<0$ if $\xi \in \mathbb{R}^{n} \backslash\{\mathbf{0}\}$, i.e. $\|\Psi\|_{\mathbf{d}}$ is a Lyapunov function for (7).

2) $\Rightarrow 4$ ) Since the origin of the system (7) is asymptotically stable then there exists a smooth d-homogeneous Lyapunov function $\tilde{V}: \mathbb{R}^{n} \rightarrow \mathbb{R}_{+}$of degree $\mu>0$. The function $V=\tilde{V}^{1 / \mu}$ is also Lyapunov function to (7) that is d-homogeneous of degree 1 , continuous at the origin and smooth outside the origin. Let us consider the map $\Psi: \mathbb{R}^{n} \rightarrow \mathbb{R}^{n}$ defined as $\Psi(\xi)=\mathbf{d}\left(\ln \left(\frac{V(\xi)}{\|\xi\|_{\mathbf{d}}}\right)\right) \xi$. Since $\frac{V(\xi)}{\|\xi\|_{\mathbf{d}}} \in \mathbb{H}_{\mathbf{d}}\left(\mathbb{R}^{n}\right), \operatorname{deg}_{\mathbf{d}}\left(\frac{V(\xi)}{\|\xi\|_{\mathbf{d}}}\right)=0$ then $\liminf _{\xi \rightarrow \mathbf{0}} \frac{V(\xi)}{\|\xi\|_{\mathbf{d}}}=$ $\inf _{\xi \in S} \frac{V(\xi)}{\|\xi\|_{\mathbf{d}}} \in(0,+\infty)$, so $\Psi$ is continuous at $\mathbf{0}$ and $\Psi(\mathbf{0})=\mathbf{0}$. Obviously, $\Psi(\mathbf{d}(s) \xi)=\mathbf{d}(s) \Psi(\xi)$ and $\|\Psi(\xi)\|_{\mathbf{d}}=V(\xi)$. We derive (10) from (3). The map $\Psi$ is homeomorphism (i.e. continuously invertible bijection) on $\mathbb{R}^{n}$. The inverse map $\Psi^{-1}: \mathbb{R}^{n} \rightarrow \mathbb{R}^{n}$ is given by $\Psi^{-1}(x)=\mathbf{d}\left(-\ln \left(\frac{V(x)}{\|x\|_{\mathbf{d}}}\right)\right) x$. Indeed, $\Psi^{-1}(\Psi(\xi))=\xi$ and $\Psi\left(\Psi^{-1}(x)\right)=x$ for all $\xi, x \in$ $\mathbb{R}^{n}$. Since $\Psi$ and $\Psi^{-1}$ are continuous at the origin, smooth outside the origin then $\Psi$ is diffeomorphism on $\mathbb{R}^{n} \backslash\{\mathbf{0}\}$.

3) $\Rightarrow$ 5) The system (8) is homogeneous of degree $\nu$ with respect to the dilation $\mathbf{d}_{0}(s)=e^{s}$ with $G_{\mathbf{d}_{0}}=I_{n}$. Note that $P_{0} G_{\mathbf{d}_{0}}+G_{\mathbf{d}_{0}}^{\top} P_{0}=2 P_{0}>0$ holds for an arbitrary symmetric positive definite matrix $P_{0}$. Taking into account $\|z\|_{\mathbf{d}_{0}}=$ $\|z\|=\sqrt{z^{\top} P_{0} z}$ we use the claim 4) to obtain the homogeneous Lyapunov function $V$ defined as $V(z)=\left\|\Psi_{0}(z)\right\|_{\mathbf{d}_{0}}^{2}=$ $\Psi_{0}^{\top}(z) P_{0} \Psi_{0}(z)$ for $z \in \mathbb{R}^{n}$. Since $\Psi_{0}$ is diffeomorphism then $\operatorname{det}\left(\frac{\partial \Psi_{0}(z)}{\partial z}\right) \neq 0$ for $z \in \mathbb{R}^{n} \backslash\{\mathbf{0}\}$. Using (6) we derive $\frac{\partial \Psi_{0}(z)}{\partial z} z=\Psi_{0}(z), \frac{\partial V}{\partial z}=2 z^{\top} \Xi^{\top}(z) P_{0} \Xi(z)$ and $\frac{\partial \Xi(z)}{\partial z_{i}} z=0$, where $\Xi(z)=\frac{\partial \Psi_{0}(z)}{\partial z}$. Finally, $\Xi\left(e^{s} z\right)=\Xi(z)$ for $z \in \mathbb{R}^{n} \backslash\{\mathbf{0}\}$ and $s \in \mathbb{R}$ due to (5) and $\operatorname{deg}_{\mathbf{d}_{0}}\left(\Psi_{0}\right)=0$.

5) $\Rightarrow$ 3) Let us consider the function $V(z)=$ $z^{\top} \Xi^{\top} P \Xi(z) z, V \in C\left(\mathbb{R}^{n}, \mathbb{R}\right) \cap C^{\infty}\left(\mathbb{R}^{n} \backslash\{\mathbf{0}\}, \mathbb{R}\right)$. Since $\frac{\partial \Xi(z)}{z}=0$ then $\frac{\partial V(z)}{\partial z}=2 z^{\top} \Xi^{\top} P \Xi(z)$ and the condition 
(11) implies that $V$ is the Lyapunov function to (8).

Therefore, the latter theorem proves two important facts:

- Any generalized homogeneous system is diffeomorphic to standard homogeneous one (see the formula (8)) and to a quadratically stable system. Indeed, making the change of variables $z=\Psi(\xi)$ we derive $\dot{z}=\tilde{f}(z)$, where $\tilde{f}(z)=\left.\frac{\partial \Psi(\xi)}{\partial \xi} f(\xi)\right|_{\xi=\Psi^{-1}(z)}$, but the criterion (10) implies that $z^{\top} P \dot{z}<0$ if $z^{\top} P z=1$, so the homogeneous norm $\|\cdot\|_{\mathbf{d}}$ is the Lyapunov function to the latter system. Finally, the change of variable $x=\|z\|_{\mathbf{d}} \mathbf{d}\left(-\ln \|z\|_{\mathbf{d}}\right) z$ gives $\|z\|_{\mathbf{d}}=\|x\|$, so the transformed system $\dot{x}=\hat{f}(x)$ is quadratically stable.

- The formula (11) gives a more constructive stability criterion. Since $\Xi(z)=\Xi\left(e^{s} z\right)$ for $z \in \mathbb{R}^{n} \backslash\{\mathbf{0}\}, s \in \mathbb{R}$ then the map $\Xi$ is constant along any straight line $\left\{e^{s} z: s \in \mathbb{R}^{n}\right\}$ if $z \neq 0$. In addition, the property $\frac{\partial \Xi(z)}{\partial z_{i}} z=0$ motivates the conjecture that a quadratic Lyapunov function ( $\Xi \equiv$ const) always exists for asymptotically stable standard homogeneous system. However, in the view of [33, Proposition 5.2] this conjecture seems to be wrong. Therefore, the condition $z^{\top} Q_{0}\left(\frac{\left(I_{n}-G_{\boldsymbol{d}}\right) z z^{\top} P}{z^{\top} P G_{\boldsymbol{d}} z}+I_{n}\right) f\left(\frac{z}{\sqrt{z^{\top} P z}}\right)<0$ if $z \neq \mathbf{0}$,
$P G_{\boldsymbol{d}}+G_{\boldsymbol{d}} P>0, \quad P>0, \quad Q_{0}>0$

derived from (11) is just sufficient stability condition.

Recall [31], [10], [11], if a homogeneous system (7) is asymptotically stable and $\operatorname{deg}_{\mathbf{d}}(f)<0$ then it is globally uniformly finite-time stable, i.e $\exists T: \mathbb{R}^{n} \rightarrow \mathbb{R}$ such that $\varphi_{x_{0}}(t)=0, \forall t>T\left(x_{0}\right)$ and $\forall x_{0} \in \mathbb{R}^{n}$.

Example 3 (Quadratic Finite-time Stability): The system $\dot{x}=A x+B u(x), A=\left(\begin{array}{ll}0 & 1 \\ 0 & 0\end{array}\right), B=\left(\begin{array}{l}0 \\ 1\end{array}\right), u(x)=\frac{k_{1} x_{1}+k_{2} x_{2} \sqrt{\sigma(x)}}{\sigma(x)}$, $\sigma(x):=q_{1}\left|x_{1}\right|^{\frac{2}{3}}+q_{2}\left|x_{2}\right|$ is $\mathbf{d}$-homogeneous of degree -1 if the dilation $\mathbf{d}$ is generated by $G_{\mathbf{d}}=\operatorname{diag}\{3,2\}$. Let us find conditions to $k_{1}, k_{2} \in \mathbb{R}, q_{1}, q_{2} \in \mathbb{R}_{+}$allowing the finite-time stability of the system. Selecting $Q_{0}=P$ in (12) we derive the stability condition $x^{\top} P \tilde{f}(x)<0$, $\tilde{f}(x)=\left(\begin{array}{c}k_{1} x_{1} \\ \frac{k_{1} x_{2}}{\tilde{N}(x)}+\frac{k_{2} x_{2}}{\sqrt{\tilde{N}(x)}}\end{array}\right), \tilde{N}(x)=\frac{q_{1}\left|x_{1}\right|^{\frac{2}{3}}\|x\|^{\frac{1}{3}}+q_{2}\left|x_{2}\right|}{\|x\|},\|x\|=$ $\sqrt{x^{\top} P x}, 0<\delta_{\min }=\min _{x^{\top} P x=1} q_{1}\left|x_{1}\right|^{\frac{2}{3}}+q_{2}\left|x_{2}\right| \leq \tilde{N}(x) \leq \delta_{\max }=$ $\max _{x^{\top} P x=1} q_{1}\left|x_{1}\right|^{\frac{2}{3}}+q_{2}\left|x_{2}\right|$. Since $x^{\top} P \tilde{f}(x)=x^{\top} P\left(\begin{array}{cc}0 & 1 \\ k_{1} & k_{2}\end{array}\right) x+$ $\frac{1-\sqrt{\tilde{N}(x)}}{\sqrt{\tilde{N}(x)}} x^{\top} P\left(\begin{array}{cc}0 & 0 \\ k_{1} & k_{2}\end{array}\right) x+\frac{1-\sqrt{\tilde{N}(x)}}{\tilde{N}(x)} x^{\top} P\left(\begin{array}{cc}0 & 0 \\ k_{1} & 0\end{array}\right) x$, then the Cauchy-Schwarz inequality implies $x^{\top} P \tilde{f}(x) \leq x^{\top} P\left(\begin{array}{cc}0 & 1 \\ k_{1} & k_{2}\end{array}\right) x$ $+\frac{|1-\tilde{N}(x)|}{\tilde{N}(x)} x^{\top} P x \leq x^{\top} P\left(\begin{array}{cc}0 & 1 \\ k_{1} & k_{2}\end{array}\right) x+\frac{\delta_{\max }-1}{\delta_{\max }} x^{\top} P x$ provided that $\left(\begin{array}{cc}0 & 0 \\ k_{1} & k_{2}\end{array}\right)^{\top} P\left(\begin{array}{cc}0 & 0 \\ k_{1} & k_{2}\end{array}\right) \leq P,\left(\begin{array}{cc}0 & 0 \\ k_{1} & 0\end{array}\right)^{\top} P\left(\begin{array}{cc}0 & 0 \\ k_{1} & 0\end{array}\right) \leq P, \delta_{\min } \geq 1$. Hence, if the system of LMIs $A X+X A^{\top}+B Y+Y^{\top} B^{\top}+\tau X<0, G_{\mathbf{d}} X+X G_{\mathbf{d}}^{\top}>0, X>0$,

$$
\left(\begin{array}{cc}
\gamma X & Y^{\top} B^{\top} \\
B Y & X
\end{array}\right)>0, \quad\left(\begin{array}{cc}
\gamma^{-1} X & E_{1} X \\
X E_{1} & X
\end{array}\right)>0, \quad E_{1}=\left(\begin{array}{ll}
1 & 0 \\
0 & 0
\end{array}\right)
$$

is feasible with respect to $X \in \mathbb{R}^{2 \times 2}, Y \in \mathbb{R}^{1 \times 2}$ for some fixed $\gamma, \tau \in(0,1)$ then the considered homogeneous ODE is finite stable with $\left(k_{1} \quad k_{2}\right)=Y X^{-1}$, and the parameters $q_{1}, q_{2} \in \mathbb{R}_{+}$selected such that $\delta_{\min } \geq(1-\tau)^{-1}$, e.g. $q_{1}=\frac{1}{(1-\tau) \sqrt[3]{e_{1}^{\top} P e_{1}}}, q_{2}=\frac{1}{(1-\tau) \sqrt{e_{2}^{\top} P e_{2}}}, e_{1}=(1,0)^{T}$, $e_{2}=(0,1)^{T}, P=X^{-1}$. In particular, for $\tau=\gamma=0.5$ MATLAB gives the following solution to the LMIs $X=\left(\begin{array}{cc}22.1245 & -6.8466 \\ -6.8466 & 9.1080\end{array}\right), Y=\left(\begin{array}{ll}-3.8858 & -3.0105\end{array}\right)$.

\section{Stabilization of Homogeneous Systems}

Let us consider the nonlinear control system

$$
\dot{x}=g(x, u) \text {, }
$$

where $x \in \mathbb{R}^{n}$ is the state vector of a plant, $g \in$ $C\left(\mathbb{R}^{n+1}, \mathbb{R}^{n}\right), g(\mathbf{0}, \mathbf{0})=\mathbf{0}$ and $u \in \mathbb{R}^{m}$ is the control input generated by a dynamic system (i.e. $u$ is a dynamic feedback)

$$
\dot{u}=k(x, u) \text {. }
$$

Based on the scheme of universal stabilizing control design given in [34], from Theorem 4 we derive a necessary and sufficient condition of existence of a generalized homogeneous map $k \in C\left(\mathbb{R}^{n+m} \backslash\{\mathbf{0}\}, \mathbb{R}^{m}\right)$ that makes the origin of the closed-loop system (13), (14) to be asymptotically stable.

Theorem 5 (On Homogeneous Dynamical Feedback):

Let $\boldsymbol{d}_{x}$ and $\boldsymbol{d}_{u}$ be dilations $\mathbb{R}^{n}$ and $\mathbb{R}^{m}$, respectively,

$$
\boldsymbol{d}:=\left(\begin{array}{cc}
\boldsymbol{d}_{x}(s) & \mathbf{0} \\
\mathbf{0} & \boldsymbol{d}_{u}(s)
\end{array}\right) \text { and } \tilde{f}=\left(\begin{array}{l}
g \\
\mathbf{0}
\end{array}\right) \in \mathbb{F}_{\boldsymbol{d}}\left(\mathbb{R}^{n+m}\right) \text {. }
$$

The origin of the system (13) is globally asymptotically stabilizable by means of the homogeneous dynamical feedback (14) with $\left(\begin{array}{c}g \\ k\end{array}\right) \in \mathbb{F}_{\boldsymbol{d}}\left(\mathbb{R}^{n+m}\right)$ if and only if there exist a number $\gamma \geq 0$, a symmetric matrix $P \in \mathbb{R}^{(n+m) \times(n+m)}$ satisfying (9) and a map $\Xi \in C^{\infty}\left(\mathbb{R}^{n} \backslash\{\mathbf{0}\}, \mathbb{R}^{n \times n}\right)$ such that $\operatorname{det}(\Xi(z)) \neq 0$, $\frac{\partial \Xi(z)}{\partial z_{i}} z=0, \Xi\left(e^{s} z\right)=\Xi(z)$ for $z \in \mathbb{R}^{n} \backslash\{\mathbf{0}\}, s \in \mathbb{R}$ and

$$
a(z)<\gamma \sqrt{b^{\top}(z) b(z)} \quad \text { for } \quad z \in S,
$$

where $a(z)=z^{\top} W(z) \tilde{f}(z), b^{\top}(z)=z^{\top} W(z)\left(\begin{array}{c}\mathbf{0} \\ I_{m}\end{array}\right), W(z)=$ $\Xi^{\top}(z) P \Xi(z)\left(\frac{\left(I_{n}-G_{\boldsymbol{d}}\right) z^{\top} z P}{z^{\top} P G_{\boldsymbol{d}} z}+I_{n}\right)$ and $S$ is the unit sphere in $\mathbb{R}^{n+m}$ with $\|z\|=\sqrt{z^{\top} P z}$. Moreover, the corresponding stabilizing homogeneous feedback law can be designed as

$$
k(\xi)=\|\xi\|_{\boldsymbol{d}}^{\operatorname{deg}_{\boldsymbol{d}}(\tilde{f})} \boldsymbol{d}_{u}\left(\ln \|\xi\|_{\boldsymbol{d}}\right) k_{0}\left(\boldsymbol{d}\left(-\ln \|\xi\|_{\boldsymbol{d}}\right) \xi\right),
$$

where $\xi=\left(\begin{array}{l}x \\ u\end{array}\right), k_{0}(z)=\left\{\begin{array}{cc}-\frac{a(z)+\sqrt{a^{2}(z)+\left(b^{\top}(z) b(z)\right)^{2}}}{b^{\top}(z) b(z)} b(z) & \text { if } b(z) \neq \mathbf{0}, \\ 0 & \text { if } b(z)=\mathbf{0},\end{array}\right.$ the homogeneous norm $\|\cdot\|_{\boldsymbol{d}}$ is induced by $\|\xi\|=\sqrt{\xi^{\top} P \xi}$.

Proof. Sufficiency. Let us show that the function $k_{0}$ is continuous on $S$. Indeed, $k_{0}(z)=$ $\frac{a(z)+\sqrt{a^{2}(z)+\left(b^{\top}(z) b(z)\right)^{2}}}{\sqrt{b^{\top}(z) b(z)}} \frac{b(z)}{\sqrt{b^{\top}(z) b(z)}}$, the first fraction is continuous and equals zero if $b(z)=\mathbf{0}$ (see, [34]), but the norm of the second fraction is globally bounded and continuous for $z \in \mathbb{R}^{n}: b(z) \neq 0$. Let us consider the closed-loop system (13), (14), (16): $\dot{\xi}=f(\xi)$, where $f=\left(\begin{array}{l}g \\ k\end{array}\right) \in \mathbb{F}_{\mathbf{d}}\left(\mathbb{R}^{n}\right)$. Under this notation, the inequality (11) becomes $a(z)+b^{\top}(z) k_{0}(z)<0$. The latter hods for for all $z \in S$. Indeed, the inequality (15) implies that $a(z)<0$ if $b(z)=\mathbf{0}$, but for $b(z) \neq \mathbf{0}$ we have $a(z)+$ $b^{\top}(z) k_{0}(z)=a(z)-b^{\top}(z) \frac{a(z)+\sqrt{a^{2}(z)+\left(b^{\top}(z) b(z)\right)^{2}}}{b^{\top}(z) b(z)} b(z)=$ $-\sqrt{a^{2}(z)+\left(b^{\top}(z) b(z)\right)^{2}}<0$. Necessity. Let us assume that there exists a map $\tilde{k} \in C\left(\mathbb{R}^{n+m}, \mathbb{R}^{m}\right)$ such that the closed-loop system (13), (14) is globally asymptotically stable and $f=\left(\begin{array}{c}g \\ \tilde{k}\end{array}\right) \in \mathbb{F}_{\mathbf{d}}\left(\mathbb{R}^{n+m}\right)$. Let $\Xi \in C^{\infty}\left(\mathbb{R}^{n+m} \backslash\{\mathbf{0}\}, \mathbb{R}^{(n+m) \times(n+m)}\right)$ be derived according to Theorem 4 such that (11) holds. To show that in this case (15) also holds for some $\gamma \geq 0$, we rewrite the inequality (11) as $a(z)<-b^{\top}(z) \tilde{k}(z)$. Since $\tilde{k} \in C\left(\mathbb{R}^{n+m}, \mathbb{R}^{m}\right)$ then $\gamma=\max _{z \in S} \sqrt{\tilde{k}^{\top}(z) \tilde{k}(z)}<+\infty$ and using 
Cauchy-Schwarz inequality we derive $-b^{\top}(z) \tilde{k}(z) \leq$ $\sqrt{\tilde{k}^{\top}(z) \tilde{k}(z)} \sqrt{b^{\top}(z) b(z)} \leq \gamma \sqrt{b^{\top}(z) b(z)}$ for $z \in S$.

For $\Xi(z)=I_{n}$ we derive from (15) the following sufficient condition of quadratic stabilizability:

$$
\begin{gathered}
z^{\top} P \tilde{f}\left(\frac{z}{\sqrt{z^{\top} P z}}\right) \leq \gamma \sqrt{z^{\top} P\left(\begin{array}{cc}
0 & 0 \\
0 & I_{m}
\end{array}\right) P z}, \quad z \neq \mathbf{0} \\
P G_{\mathbf{d}}+G_{\mathbf{d}}^{\top} P>0, \quad P>0 .
\end{gathered}
$$

Example 4 (Dynamical Stabilization in a Finite Time):

Let us consider the stabilization problem for the system $\dot{x}_{1}=\left|x_{1}\right|^{\frac{1}{3}} u+x_{2}, \dot{x}_{2}=u$, where $x_{1}, x_{2}, u \in \mathbb{R}$. The vector field $\tilde{f}: \mathbb{R}^{3} \rightarrow \mathbb{R}^{3}$ given by $\tilde{f}(z)=\left(\begin{array}{c}\left|z_{1}\right|^{\frac{1}{3}} z_{3}+z_{2} \\ z_{3} \\ 0\end{array}\right)$, $z=\left(z_{1}, z_{2}, z_{3}\right)^{\top}=\left(x_{1}, x_{2}, u\right)$ is d-homogeneous of degree -1 with respect to the dilation $\mathbf{d}$ in $\mathbb{R}^{3}$ generated by $G_{\mathbf{d}}=\operatorname{diag}\{3,2,1\}$. Hence, the sufficient stabilizability condition (17) becomes $z^{\top} P A(z) z<\gamma \sqrt{z^{\top} P z} \sqrt{z^{\top} P e_{3} e_{3}^{\top} P z}, P G_{\mathbf{d}}+G_{\mathbf{d}}^{\top} P>0$, $P>0$, where $A(z)=\left(\begin{array}{ccc}0 & 1 & \nu(z) \\ 0 & 0 & 1 \\ 0 & 0 & 0\end{array}\right), \nu(z)=\left(\frac{\left|z_{1}\right|}{\sqrt{z^{\top} P z}}\right)^{\frac{1}{3}}$. Let $X \in \mathbb{R}^{3 \times 3}$ and $\gamma_{0}>0$ satisfy the linear matrix inequalities $X>0, X A_{i}+A_{i}^{\top} X-2 \gamma_{0} e_{3} e_{3}^{\top}<0, X G_{\mathbf{d}}+G_{\mathbf{d}} X>0$, $i=0,1, A_{i}=\left(\begin{array}{lll}0 & 1 & i \\ 0 & 0 & 1 \\ 0 & 0 & 0\end{array}\right)$. Since $A(z)=\alpha(z) A_{1}+(1-\alpha(z)) A_{0}$, where

$\alpha(z)=\left(\frac{\left|z_{1}\right|}{\sqrt{z^{\top} P z}}\right)^{\frac{1}{3}}$ and $\alpha(z) \in[0,1]$ provided that $p_{11}=1$, then for $P=\tilde{P} /\left(\tilde{p}_{11}\right), \tilde{P}=X^{-1}$ the stabilizability condition (17) holds. So, the considered system can be stabilized in a finite time (due to $\operatorname{deg}_{\mathbf{d}}(\tilde{f})=-1$ ) to zero by means of the d-homogeneous dynamical feedback $\dot{u}=$ $k_{0}\left(\frac{z}{\|z\|_{\mathrm{d}}}\right)$, where $k_{0}(z)=\left\{\begin{array}{cc}-\frac{a(z)+\sqrt{a^{2}(z)+b^{4}(z)}}{b(z)} & \text { if } b(z) \neq 0, \\ 0 & \text { if } b(z)=0,\end{array}\right.$ $a(z)=z^{\top} P \tilde{f}(z)$ and $b(z)=p_{13} z_{1}+p_{23} z_{2}+p_{33} z_{3}$.

\section{Discussions AND CONCLUSIONS}

It is proven that any asymptotically stable generalized homogeneous system is equivalent (diffeomorphic) to quadratically stable one. The necessary and sufficient condition for quadratic-like stability (stabilizability) of homogeneous (control) system is derived. On the examples, it is demonstrated that this condition may simplify, in some particular cases, design of finite-time stabilizing feedback laws. For the general case an appropriate computational procedure is needed to be developed for Lyapunov function design based on (11). This is the important problem for future research.

\section{REFERENCES}

[1] D. J. Gross, "The role of symmetry in fundamental physics," Proceeding of the National Academy of Sciences of The United States of America, vol. 93, no. 25, pp. 14256-14259, 1996.

[2] V. Zubov, "On systems of ordinary differential equations with generalized homogenous right-hand sides," Izvestia vuzov. Mathematica., vol. 1, pp. 80-88, 1958 (in Russian).

[3] H. Hermes, "Nilpotent approximations of control systems and distributions," SIAM Journal of Control and Optimization, vol. 24, no. 4, p. $731,1986$.

[4] M. Kawski, "Geometric homogeneity and stabilization," in Proc. IFAC Nonlinear Control Symposium, A. Krener and D. Mayne, Eds., Lake Tahoe, CA, 1995, pp. 164-169.

[5] L. Rosier, "Etude de quelques problemes de stabilization," PhD Thesis, Ecole Normale Superieure de Cachan (France), 1993.

[6] R. Sepulchre and D. Aeyels, "Homogeneous Lyapunov Functions and Necessary Conditions for Stabilization," Mathematics of Control, Signals and Systems, vol. 9, pp. 34-58, 1996.
[7] V. I. Zubov, Methods of A.M. Lyapunov and Their Applications. Noordhoff, Leiden, 1964

[8] A. Andreini, A. Bacciotti, and G. Stefani, "Global stabilizability of homogenenous vector fields of odd degree," Systems \& Control Letters, vol. 10, pp. 251-256, 1988.

[9] J.-M. Coron and L. Praly, "Adding an integrator for the stabilization problem," Systems \& Control Letters, vol. 17, no. 2, pp. 89-104, 1991.

[10] Y. Orlov, "Finite time stability and robust control synthesis of uncertain switched systems," SIAM Journal of Control and Optimization, vol. 43, no. 4, pp. 1253-1271, 2005.

[11] A. Levant, "Homogeneity approach to high-order sliding mode design," Automatica, vol. 41, no. 5, pp. 823-830, 2005.

[12] L. Rosier, "Homogeneous Lyapunov function for homogeneous continuous vector field," Systems \& Control Letters, vol. 19, pp. 467-473, 1992.

[13] V. Andrieu, L. Praly, and A. Astolfi, "Homogeneous Approximation, Recursive Observer Design, and Output Feedback," SIAM Journal of Control and Optimization, vol. 47, no. 4, pp. 1814-1850, 2008.

[14] D. Efimov and W. Perruquetti, "Oscillations conditions in homogenous systems," in Proc. IFAC NOLCOS Symp., 2010, pp. 1379-1384.

[15] A. Polyakov, D. Efimov, and W. Perruquetti, "Robust stabilization of mimo systems in finite/fixed time," International Journal of Robust and Nonlinear Control, vol. 26, no. 1, pp. 69-90, 2016.

[16] K. Zimenko, D. Efimov, A. Polyakov, and W. Perruquetti, "A note on delay robustness for homogeneous systems with negative degree," Automatica, vol. 79, no. 5, pp. 178-184, 2017.

[17] J.-B. Pomet and C. Samson, "Time-varying exponential stabilization of nonholonomic systems in power form," Inria, Tech. Rep., 1993.

[18] A. Polyakov, D. Efimov, E. Fridman, and W. Perruquetti, "On homogeneous distributed parameters equations," IEEE Transactions on Automatic Control, vol. 61, no. 11, pp. 3657-3662, 2016.

[19] V. V. Khomenuk, "On systems of ordinary differential equations with generalized homogenous right-hand sides," Izvestia vuzov. Mathematica., vol. 3(22), pp. 157-164, 1961 (in Russian).

[20] I. Karafyllis and Z.-P. Jiang, Stability and Stabilization of Nonlinear Systems. Springer, 2011.

[21] A. Poznyak, A. Polyakov, and V. Azhmyakov, Attractive Ellipsoids in Robust Control. Birkhauser, 2014.

[22] H. Hermes, "Homogeneous feedback controls for homogeneous systems," Systems \& Control Letters, vol. 24, pp. 7-11, 1995.

[23] L. Praly, "Generalized weighted homogeneity and state dependent time scale for linear controllable systems," in Conference on Decision and Control, 1997, pp. 4342-4347.

[24] L. Grune, "Homogeneous state feedback stabilization of homogeneous systems," SIAM Journal of Control and Optimization, vol. 38, no. 4, pp. 1288-1308, 2000.

[25] N. Nakamura, H. Nakamura, and Y. Yamashita, "Homogeneous stabilization for input-affine homogeneous systems," in Conference on Decision and Control, 2007, pp. 80-85.

[26] W. Perruquetti, T. Floquet, and E. Moulay, "Finite-time observers: application to secure communication," IEEE Transactions on Automatic Control, vol. 53, no. 1, pp. 356-360, 2008.

[27] S. Boyd, E. Ghaoui, E. Feron, and V. Balakrishnan, Linear Matrix Inequalities in System and Control Theory. Philadelphia: SIAM, 1994.

[28] A. Polyakov, J.-M. Coron, and L. Rosier, "On finite-time stabilization of evolution equations: A homogeneous approach," in Conference on Decision and Control, 2016, pp. 3143-3148.

[29] A. Pazy, Semigroups of Linear Operators and Applications to Partial Differential Equations. Springer, 1983.

[30] R. Courant and F. John, Introduction to calculus and analysis (Vol. II/1). New York: Springer, 2000.

[31] S. P. Bhat and D. S. Bernstein, "Geometric homogeneity with applications to finite-time stability," Mathematics of Control, Signals and Systems, vol. 17, pp. 101-127, 2005.

[32] E. Moulay and W. Perruquetti, "Finite-time stability of differential inclusions," IMA Journal Mathematical Control and Information Sciences, vol. 22(4), pp. 465-475, 2005.

[33] A. Bacciotti and L. Rosier, Lyapunov Functions and Stability in Control Theory. Springer, 2001.

[34] E. Sontag, "A 'universal' construction of artstein's theorem on nonlinear stabilization," Systems \& Control Letters, vol. 13, pp. 117-123, 1989. 